\title{
Existence of Quadrature Formulae with Almost Equal Weights*
}

\author{
By K. Salkauskas
}

\begin{abstract}
The condition that an interpolatory quadrature formula on $\boldsymbol{n}$ nodes have degree of precision at least $n$ and positive weights defines a homeomorphism between the sets of admissible nodes and weights of such formulae for each $n$. This is used to prove that the only formulae having "almost equal" weights are the Chebyshev formulae.
\end{abstract}

1. Introduction. It is well known that for many weight functions it is not possible to construct Chebyshev quadrature formulae of high order with real nodes. For example, with the weight function $w=1$, nonreal nodes appear for $n=8$ and $n>9$, where $n$ is the number of nodes. On the other hand, if $w(x)=\left(1-x^{2}\right)^{-1 / 2}$, then the corresponding Chebyshev formulae on $[-1,1]$ exist for all positive integers $n$. In those cases where the (equally weighted) Chebyshev formulae do not exist we may ask whether it is possible to select real nodes in such a way that the weights are almost equal in some sense, while the formula retains the degree of precision of the Chebyshev case. For example, Ostrowski [2] has asked for the smallest constant $C_{n} \geqq 1$ such that there exists an $n$-point quadrature formula whose weights have ratios less than or equal to $C_{n}$. Alternatively, one may choose to employ the sum of squared weights as a measure of the equality of weights. When the weights are equal (to each other), the sum of their squares attains its absolute minimum given that the sum of the weights is a positive constant, say $\alpha_{0}$; the last condition is independent of nodes and expresses the requirement that the formula be exact for a constant function. However, in general the weights are functions of the nodes and one may wonder whether the sum of squared weights as a function of the nodes can have other local minima. What both the above-mentioned approaches to the problem have in common is this. Let the weights of an $n$-point quadrature formula be $h_{1}, \cdots, h_{n}$. Let $h=\left(h_{1}, \cdots, h_{n}\right)$. We have $\sum h_{i}=\alpha_{0}$, a constant. Restricting ourselves to formulae with positive weights, we let $\beta=\left\{h: \sum h_{i}=\alpha_{0}, h_{i}>0\right\}$. Let a continuous nonnegative function $\Phi$ be defined on $\odot$ with the property that $\Phi$ has one, and only one, minimum on $\odot$ at the point $h=\left(\alpha_{0} / n, \cdots, \alpha_{0} / n\right)$. Denote the nodes by $x_{1}, \cdots, x_{n}$, and let $x=\left(x_{1}, \cdots, x_{n}\right)$. Then $h=\xi(x)$, and $\Phi(h)=\Phi^{*}(x)$. We ask whether $\Phi^{*}$ has local minima for admissible real points $x$. It may not be obvious that Ostrowski's proposal leads to the consideration of such a function $\Phi$; this will be dealt with in the last section. We shall prove the following

THEOREM. The only n-point quadrature formulae which are exact for $f_{i}(x)=x^{i}$

Received October 24, 1969, revised March 9, 1970 and April 27, 1970.

AMS 1970 subject classifications. Primary 41A55.

Key words and phrases. Chebyshev quadrature, almost equally weighted quadrature.

* This research was supported in part by the National Research Council of Canada, under Grant No. A-4050.

Copyright $\odot$ 1971, American Mathematical Society 
$(j=0,1, \cdots, n)$, have positive weights, nodes in $(-1,1)$, and a local minimum value of $\Phi^{*}$, are the Chebyshev formulae.

The next section contains the definitions needed to make the terminology of this section precise, as well as some well-known results that will be needed in Section 3 for the proof of the Theorem.

2. Interpolatory $n$-Point Formulae. Let $f_{i}(t)=t^{i}(j=0,1, \cdots)$, and let $w$ be a nonnegative function that is Riemann-integrable on $[-1,1]$ in either the proper or improper sense, and whose integral over $[-1,1]$ is positive. We call $w$ a weight function. In what follows, $w$ will be considered fixed. For a fixed positive integer $n$ and for any function $f$ that is defined on $(-1,1)$ and such that $w f$ is integrable on $[-1,1]$, we define two linear functionals $I$ and $Q_{n}$ by

$$
I(f) \equiv \int_{-1}^{1} w f, \quad Q_{n}(f) \equiv \sum_{i=1}^{n} h_{i} f\left(x_{i}\right)
$$

In $Q_{n}$ the weights $h_{i}$ are nonzero, and the nodes $x_{i}$ are real, distinct and lie in $(-1,1)$. The weights and nodes are parameters whose values determine how well $Q_{n}(f)$ approximates $I(f) . Q_{n}(f)$ is an $n$-point quadrature formula intended to approximate $I(f)$ and is interpolatory if, and only if

$$
Q_{n}\left(f_{i}\right)=I\left(f_{j}\right), \quad(j=0,1, \cdots, n-1) .
$$

This can be accomplished for an arbitrary set of real and distinct nodes by setting

$$
h_{i}=\frac{1}{\pi^{\prime}\left(x_{i}\right)} \int_{-1}^{1} \frac{w(t) \pi(t)}{t-x_{i}} d t, \quad(i=1,2, \cdots, n),
$$

where $\pi(t)=\left(t-x_{1}\right)\left(t-x_{2}\right) \cdots\left(t-x_{n}\right)$. If we set $I\left(f_{i}\right)=\alpha_{i}$, then (2.1) becomes

$$
\sum_{i=1}^{n} h_{i} x_{i}^{i}=\alpha_{i}, \quad(j=0,1, \cdots, n-1) .
$$

For any specific set of nodes and corresponding weights given by (2.2) we define the degree of precision of $Q_{n}$ to be the smallest integer $d$ such that

$$
Q_{n}\left(f_{d+1}\right) \neq I\left(f_{d+1}\right) \text {. }
$$

Since $Q_{n}$ is interpolatory, $d \geqq n-1$. It is also well known that the Gauss formulae have $d=2 n-1$, and that $d$ cannot be increased. Hence $n-1 \leqq d \leqq 2 n-1$. Chebyshev formulae have degree of precision at least $n$, and this is a condition that we shall impose on $Q_{n}$. Hence the nodes and weights of $Q_{n}$ must satisfy the equations

$$
\sum_{i=1}^{n} h_{i} x_{i}^{i}=\alpha_{i} \quad(j=0,1, \cdots, n) .
$$

Let $x=\left(x_{1}, \cdots, x_{n}\right)$, and let

$$
S_{n}=\left\{x: I(\pi)=0, x_{1}<x_{2}<\cdots<x_{n}\right\} .
$$

To every $x \in S_{n}$ there corresponds an interpolatory quadrature formula whose weights we may obtain from (2.2), and the condition $I(\pi)=0$ is necessary and sufficient for the formula to have degree of precision $n$. We shall be concerned with formulae having positive weights, and therefore restrict $x$ to a subset $X$ of $S_{n}$. It is easy to see 
from (2.2) and the fact that $x_{1}<x_{2}<\cdots<x_{n}$ for $x \in S_{n}$, that $h_{i}>0$ (i=1, $2, \cdots, n)$, if and only if

$$
(-1)^{n-i} \int_{-1}^{1} w(t) \pi(t) /\left(t-x_{i}\right) d t>0, \quad(i=1,2, \cdots, n) .
$$

We define $x$ to be that subset of $S_{n}$ in which (2.5) holds and in which $-1<x_{1}$, $x_{n}<1$. Then $x$ is open in $S_{n}$, and every point of $X$ is an interior point. $x$ is not empty since the nodes of a Gaussian formula corresponding to the weight function $w$ satisfy the requirements of $x$.

Let $\left(h_{1}, \cdots, h_{n}\right)=h$ denote the set of weights of $Q_{n}$, with $h_{i}$ associated with $x_{i}$. Let $P=\left\{h: \sum h_{i}=\alpha_{0}, h_{i}>0\right\}$. Clearly, Eqs. (2.2) or their equivalent (2.1) define a mapping $\xi$ of $X$ into $P$. In fact, for each $x \in X$ there is determined a unique $h \in P$. Let $\mathfrak{H C}=\xi(x)$. Then $\xi$ maps $X$ onto $\mathcal{H}$.

\section{Proof of the Theorem.}

LEMma 1. If $x, y \in x$, and $\xi(x)=\xi(y)$, then $x=y$.

Proof. The hypotheses of the Lemma imply that

$$
\sum_{i=1}^{n} h_{i} f\left(x_{i}\right)=\sum_{i=1}^{n} h_{i} f\left(y_{i}\right)
$$

for all polynomials $f$ of degree $\leqq n$. We shall construct a polynomial of degree $\leqq n$ for which the equality does not hold when $x \neq y$.

Let $s_{i}=x_{i}-y_{i}(i=1,2, \cdots, n)$, and consider the sequence $\left\{s_{i}\right\}_{1}^{n}$. Not more than $n-1$ sign changes can occur between the terms of the sequence. From $\left\{s_{i}\right\}_{1}^{n}$ extract a sequence $\left\{t_{i}\right\}_{1}^{p}$ by deleting all zero terms of $\left\{s_{i}\right\}_{1}^{n}$ and replacing every subsequence of terms with the same sign by the first term of that subsequence. The resulting sequence $\left\{t_{i}\right\}_{1}^{p}$ has at least one term if $x \neq y$, but not more than $n$, so $1 \leqq$ $p \leqq n$. Now, if $p>1, t_{m}=x_{r}-y_{r}>0$ and $t_{m+1}=x_{s}-y_{c}<0$, select $\xi_{m} \in\left[x_{s-1}, x_{s}\right]$. If $t_{m}<0$ and $t_{m+1}>0$, select $\xi_{m} \in\left[y_{s-1}, y_{s}\right]$. Let this be done for the whole sequence $\left\{t_{i}\right\}_{1}^{p}$. Let

$$
\begin{aligned}
f^{\prime}(t) & =(-1)^{p-1} \operatorname{sgn} t_{1} \prod_{i=1}^{p-1}\left(t-\xi_{i}\right), & & p>1, \\
& =\operatorname{sgn} t_{1}, & & p=1 .
\end{aligned}
$$

Then

$$
f(t) \equiv \int_{0}^{t} f^{\prime}(\tau) d \tau
$$

is a polynomial of degree $p \leqq n$. It has the property that $f\left(x_{i}\right) \geqq f\left(y_{i}\right)$, with equality only if $x_{i}=y_{i}$. Since $x, y \in x$, we have $h_{i}>0$. Hence

$$
\sum_{i=1}^{n} h_{i} f\left(x_{i}\right)>\sum_{i=1}^{n} h_{i} f\left(y_{i}\right), \quad x \neq y .
$$

This is a contradiction.

It will be convenient to abbreviate Eqs. (2.3) as

$$
F_{j}(x ; h)=0 \quad(j=0,1, \cdots, n) .
$$


The equation $F_{0}(x ; h)=0$ is part of the definition of $\mathfrak{H C}$ and does not involve the nodes. We now prove

LEMMA 2. The mapping $\xi: x \rightarrow$ TC is bicontinuous.

Proof. For every $x \in X, h=\xi(x) \in \mathcal{H}$ may be defined by the equations $F_{i}(x ; h)=0(j=0,1, \cdots, n-1)$. The Jacobian

$$
\frac{\partial\left(F_{0}, F_{1}, \cdots, F_{n-1}\right)}{\partial\left(h_{1}, h_{2}, \cdots, h_{n}\right)} \equiv V
$$

where $V$ is the Vandermonde determinant of the nodes, and does not vanish on $X \times \mathfrak{H}$. Hence $\xi$ is continuous. Also

$$
\frac{\partial\left(F_{1}, F_{2}, \cdots, F_{n}\right)}{\partial\left(x_{1}, x_{2}, \cdots, x_{n}\right)}=n ! V \prod_{i=1}^{n} h_{i} \neq 0, \quad(x ; h) \in X \times \mathfrak{H} .
$$

If, for $h \in \mathfrak{K}$, we consider $\xi^{-1}$ to be defined implicitly by the last $n$ of the Eqs. (2.3), then the above implies that $\xi^{-1}$ is continuous, and $\xi$ is a homeomorphism of $X$ onto $\mathcal{H}$.

Brouwer's Invariance of Domain Theorem [3] can be employed to show that every point of $\mathfrak{F}=\xi(x)$ is an interior point relative to $\odot$, for the condition $I(\pi)=0$ is equivalent to

$$
\alpha_{n}+\alpha_{n-1} a_{1}+\cdots+\alpha_{0} a_{n}=0,
$$

where $\pi(t)=t^{n}+a_{1} t^{n-1}+\cdots+a_{n}$. We identify $\pi(t)$ with the point $\left(a_{1}, \cdots, a_{n}\right) \in$ $\mathbb{R}^{n}$, and (3.1) defines a hyperplane $Q_{1}$ in $R^{n}$. This identification represents a homeomorphic map $\psi$ of $S_{n}$ into $Q_{1}$, and the image of $S_{n}$ is open in $Q_{1}$. Since $X$ is open relative to $S_{n}, \psi(x)$ is open relative to $\psi\left(S_{n}\right) \subset Q_{1}$. Hence $\psi(x)$ is open in $Q_{1}$. Now $\mathfrak{H C}$ is a homeomorphic image of $\psi(\mathscr{X})$ under $\xi \psi^{-1}$ and is a subset of the set $\beta$ contained in the hyperplane $Q_{2}: \sum_{i=1}^{n} h_{i}=\alpha_{0}$. Hence, by Brouwer's Theorem, $\mathfrak{F}$ is open in $Q_{2}$. But $\beta$ is also open in $Q_{2}$, so that $\mathcal{H C}$ is open in $P$.

If we now consider the function $\Phi$ on $\odot$, having its only local minimum at $\bar{h}=$ $\left(\alpha_{0} / n, \cdots, \alpha_{0} / n\right)$, then, if $\bar{h} \notin \mathcal{H}$, the problem of minimizing $\Phi$ as a function of the nodes, i.e. $\Phi^{*}$, has no solution in $X$. But the statement that $\bar{h} \in \mathcal{H}$, implies the existence of the Chebyshev formula with real nodes. This proves the theorem.

4. Ostrowski's Proposal. Consider $h \in P$. Let $\Phi(h)=\max _{i, j} h_{i} / h_{i}$. The constant $C_{n}$ required is

$$
C_{\mathrm{n}} \equiv \min _{h \in \Re C} \Phi(h),
$$

if it exists. Clearly $\min _{h \in \mathcal{Q}} \Phi(h)=1$ and occurs at $h=\bar{h}=\left(\alpha_{0} / n, \cdots, \alpha_{0} / n\right)$.

Suppose that $\Phi$ has another local minimum at $h=h^{*} \neq \bar{h}$. Then $\Phi\left(h^{*}\right)>1$. Therefore, we can order the components of $h^{*}$ as follows:

$$
h_{i_{1}}^{*} \geqq h_{i_{2}}^{*} \geqq \cdots \geqq h_{i_{\mu}}^{*}>h_{i_{1}}^{*} \geqq h_{i_{2}}^{*} \geqq \cdots \geqq h_{i_{\lambda}}^{*}>0, \quad \mu+\lambda=n .
$$

Now let $\epsilon>0$ be a number $<\frac{1}{2}\left(h_{i \mu}^{*}-h_{i_{1}}^{*}\right)$. Consider the point $h^{\prime}$ with coordinates obtained from those of $h^{*}$ via

$$
h_{i_{k}}^{\prime}=h_{i_{k}}^{*}-\frac{\epsilon}{\mu}, \quad h_{j_{l}}^{\prime}=h_{j_{l}}^{*}+\frac{\epsilon}{\lambda}, \quad(k=1, \cdots, \mu ; l=1, \cdots, \lambda) .
$$


Then $h^{\prime} \in \rho$, and

$$
\Phi\left(h^{\prime}\right)=\frac{h_{i_{1}}^{\prime}}{h_{i_{\lambda}}^{\prime}}=\frac{h_{i_{1}}^{*}-\epsilon / \mu}{h_{i_{\lambda}}^{*}+\epsilon / \lambda}<\frac{h_{i_{1}}^{*}}{h_{i_{\lambda}}^{*}}=\Phi\left(h^{*}\right) .
$$

Hence every neighbourhood of $h^{*}$ contains points $h^{\prime}$ at which $\Phi\left(h^{\prime}\right)<\Phi\left(h^{*}\right)$. It follows that $\Phi$ has one, and only one minimum, at $\bar{h}$, and if $\bar{h} \notin \mathcal{H}$, then no smallest $C_{n}$ can be found.

The results presented here are not valid if the degree of precision is not required to be as great as $n$, for then, if $d$ is only required to be $n-1, \xi$ is no longer a homeomorphism. If $d>n$, then, in view of the additional constraints imposed on the nodes, it may be possible to obtain interpolatory quadrature formulae which minimize $\Phi$ and have real nodes. It may also be of interest to consider those cases in which $x_{1}=-1$ or $x_{n}=1$.

The University of Calgary

Calgary 44

Alberta, Canada

1. V. I. KrYlov, Approximate Calculation of Integrals, Fizmatgiz, Moscow, 1959; English transl., Macmillan, New York, 1962. MR 22 \#2002; MR 26 \#2008.

2. A. M. Ostrowski, On Trends and Problems in Numerical Approximation, Proc. Sympos. Numerical Approximation, Univ. of Wisconsin Press, Madison, Wis., 1959.

3. W. Hurewicz \& H. Wallman, Dimension Theory, Princeton Math. Series, vol. 4, Princeton Univ. Press, Princeton, N. J., 1941. MR 3, 312. 